\title{
Evolution of ion bunch profile in the presence of longitudinal coherent electron cooling
}

\author{
G. Wang \\ Collider-Accelerator Department, Brookhaven National Laboratory, Upton, \\ New York 11973-5000, USA
}

(Received 8 May 2019; published 25 November 2019)

\begin{abstract}
In the presence of longitudinal coherent electron cooling, the evolution of the line-density profile of a circulating ion bunch can be described by the 1D Fokker-Planck equation. We show that, in the absence of diffusion, the 1D equation can be solved analytically for a particular dependence of the cooling force on the synchrotron amplitude. For more general cases with arbitrary diffusion, we solved the 1D Fokker-Planck equation numerically, and the numerical solutions have been compared with results from macroparticle tracking.
\end{abstract}

DOI: 10.1103/PhysRevAccelBeams.22.111002

\section{INTRODUCTION}

The future electron-ion collider (EIC) demands a strong hadron cooling technique to reach the luminosity level where all the relevant physics can be fully covered. ${ }^{1}$ As a potential candidate to providing such a cooling technique, the concept of coherent electron cooling $(\mathrm{CeC})$ and its variants have been extensively investigated [3-7]. Both analytical and simulation tools have been developed to predict the ion bunch evolution in the presence of $\mathrm{CeC}$, which is essential for diagnosing as well as optimizing the cooling system $[8,9]$. While simulation through macroparticle tracking is the most straightforward approach in predicting the ion bunch evolution under cooling, analytical tools are needed in both benchmarking the simulation code and providing a fast estimate for the ion bunch profile.

In the case that the system has only longitudinal cooling, the evolution of the ion bunch under cooling can be described by the 1D Fokker-Planck equation. Analytical tools are being developed to solve the 1D Fokker-Planck equation with a given cooling rate and diffusion coefficient. We review the 1D Fokker-Planck equation and its equilibrium solutions in Sec. II. In Sec. III, we derived an

\footnotetext{
${ }^{1}$ There are currently two designs of a future EIC, the BNL-EIC (eRHIC) and JLEIC. For BNL-EIC, the goal of strong hadron cooling is to counteract the emittance growth due to intrabeam scattering (IBS) which has a growth time of about 2 hours [1]. For JLEIC, strong hadron cooling is required to reduce $3 \mathrm{D}$ emittance of the hadron beam up to 1 order of magnitude [2].

gawang@bnl.gov

Published by the American Physical Society under the terms of the Creative Commons Attribution 4.0 International license. Further distribution of this work must maintain attribution to the author(s) and the published article's title, journal citation, and DOI.
}

analytical solution of the 1D Fokker-Planck equation for a specific dependence of the cooling rate on the synchrotron oscillation amplitude, in the limit of the vanishing diffusion coefficient. Section IV contains our approach of numerically solving the 1D Fokker-Planck equation for finite diffusion coefficient and arbitrary dependence of the cooling rate on the synchrotron oscillation amplitude. Results show that the numerical solution agrees well with the analytical solution at the proper limits taken by the latter. In Sec. V, we present the numerical solution for parameters of the proof of the $\mathrm{CeC}$ principle experiment and compare it with that obtained from macroparticle tracking in Sec. VI. We provide a summary in Sec. VII.

\section{1D FOKKER-PLANCK EQUATION}

In the cooling section of a $\mathrm{CeC}$ system, a circulating ion experiences a coherent energy kick induced by itself to correct its energy error as well as a random energy kick induced by its neighbors (electrons and ions). In addition, the circulating ions also receive random kicks from intrabeam scattering (IBS). In the presence of the cooling force and random diffusive kicks, the evolution of the longitudinal phase space density is described by the 1D FokkerPlanck equation $[10,11]$

$$
\begin{aligned}
& \frac{\partial}{\partial t} F(I, t)-\frac{\partial}{\partial I}[\zeta(I) \cdot I \cdot F(I, t)]-\frac{\partial}{\partial I}\left(I \cdot D(I) \cdot \frac{\partial F(I, t)}{\partial I}\right) \\
& \quad=0,
\end{aligned}
$$

where $F(I, t)$ is the longitudinal phase space density averaged over one synchrotron oscillation, $I$ is the amplitude of the synchrotron oscillation, and $\zeta(I)=-\frac{1}{I}\left\langle\frac{\Delta I_{c}}{T_{\text {rev }}}\right\rangle_{T_{s}}$ and $D(I)=\frac{1}{I} \frac{\left\langle\Delta I_{d}^{2}\right\rangle_{T_{S}}}{2 T_{\mathrm{rev}}}$ are the cooling rate and diffusion 
coefficient averaged over one synchrotron oscillation. The diffusion coefficients are to be calculated from the summation of all random kicks. Throughout the context, we assume that the ion bunch length is much smaller than the fundamental wavelength of the rf cavity; consequently, we can apply a small amplitude approximation for the synchrotron oscillation. The action-angle variables under this approximation are given by [12]

$$
P=\sqrt{2 I} \cos w
$$

and

$$
\phi=\sqrt{2 I} \sin w,
$$

where $\phi$ is the rf phase and

$$
P \equiv-h \frac{|\eta|}{\nu_{s}} \frac{\Delta p}{p}
$$

is the normalized energy deviation of the ion. The unperturbed motion of the ions can be derived from the Hamiltonian

$$
H_{0}=\frac{1}{2} \omega_{0} \nu_{s} P^{2}+\omega_{0} \nu_{s} \frac{1}{2} \phi^{2}=\omega_{0} \nu_{s} I,
$$

and the distribution function, $F(I, t)$, satisfies the following relation:

$$
\int_{0}^{\infty} F(I, t) d I=\frac{N}{2 \pi},
$$

with $N$ representing the total number of ions in the bunch. It follows from Eqs. (2), (3), and (6) that

$$
\begin{aligned}
\int_{-\infty}^{\infty} F\left(\frac{P^{2}+\phi^{2}}{2}, t\right) d P d \phi & =\int_{-\infty}^{\infty} F\left(\frac{P^{2}+\phi^{2}}{2}, t\right) \frac{\partial(P, \phi)}{\partial(I, w)} d I d w \\
& =\int_{0}^{2 \pi} \int_{0}^{\infty} F(I, t) d I d w \\
& =N
\end{aligned}
$$

hence, the distribution function in canonical variable, $(\phi, P)$, is given by

$$
K(P, \phi, t)=F\left(\frac{1}{2} P^{2}+\frac{1}{2} \phi^{2}, t\right) .
$$

At equilibrium, the first term on the lhs of Eq. (1) vanishes, and we obtain the following solution for the equilibrium distribution of the ion beam:

$$
F_{\text {eq }}(I)=A \exp \left\{-\int \frac{\zeta(I)}{D(I)} d I\right\}
$$

\section{ANALYTICAL SOLUTIONS IN THE LIMIT OF ZERO DIFFUSION}

If the expected energy kicks from diffusion are much smaller than that from the coherent cooling kicks, i.e., $\langle D(I)\rangle \ll\langle\xi(I) I\rangle$, with the angled bracket denoting averaging over all ions, we can neglect the third term on the lhs of Eq. (1) and obtain

$$
\frac{\partial}{\partial t} \tilde{F}(I, t)-\alpha(I) \frac{\partial}{\partial I} \tilde{F}(I, t)=0,
$$

with

$$
\tilde{F}(I, t) \equiv \zeta(I) \cdot I \cdot F(I, t)
$$

and

$$
\alpha(I) \equiv I \cdot \zeta(I)
$$

The solution of Eq. (10), which satisfies the initial condition of $\tilde{F}\left(I, t_{0}\right)=\tilde{F}_{0}(I)$, reads (see Appendix A)

$$
F(I, t)=\frac{h^{-1}(C) \zeta\left[h^{-1}(C)\right] F_{0}\left[h^{-1}(C)\right]}{\zeta(I) \cdot I},
$$

where

$$
C=t-t_{0}+\int \frac{d I}{\alpha(I)}
$$

and

$$
h(I) \equiv \int \frac{d I}{\alpha(I)}
$$

Equation (13) is a general result valid for any given form of cooling rate, $\zeta(I)$. In practice, however, a closed-form solution of $h^{-1}(C)$ does not always exist for the specific $\zeta(I)$, and consequently, the inverse function must be found numerically. As an analytically tractable example, we assume the dependence of the cooling rate on the synchrotron oscillation action takes the following form:

$$
\zeta(I)=\zeta_{0} \frac{I_{e}}{I+I_{e}},
$$

where $\zeta_{0}$ is the cooling rate for ions with a near-zero synchrotron oscillation amplitude, and $I_{e}$ is a parameter determining the effective cooling range in the longitudinal direction. ${ }^{2}$ Inserting Eqs. (16) and (12) into Eq. (15) yields

\footnotetext{
${ }^{2} I_{e}$ is determined by the bunch length of the electron beam [see Eq. (28)], which can be significantly shorter than that of the ion beam. For the proof of the $\mathrm{CeC}$ principle experiment at RHIC, the electron bunch is more than 2 orders of magnitude shorter than the ion bunch.
} 

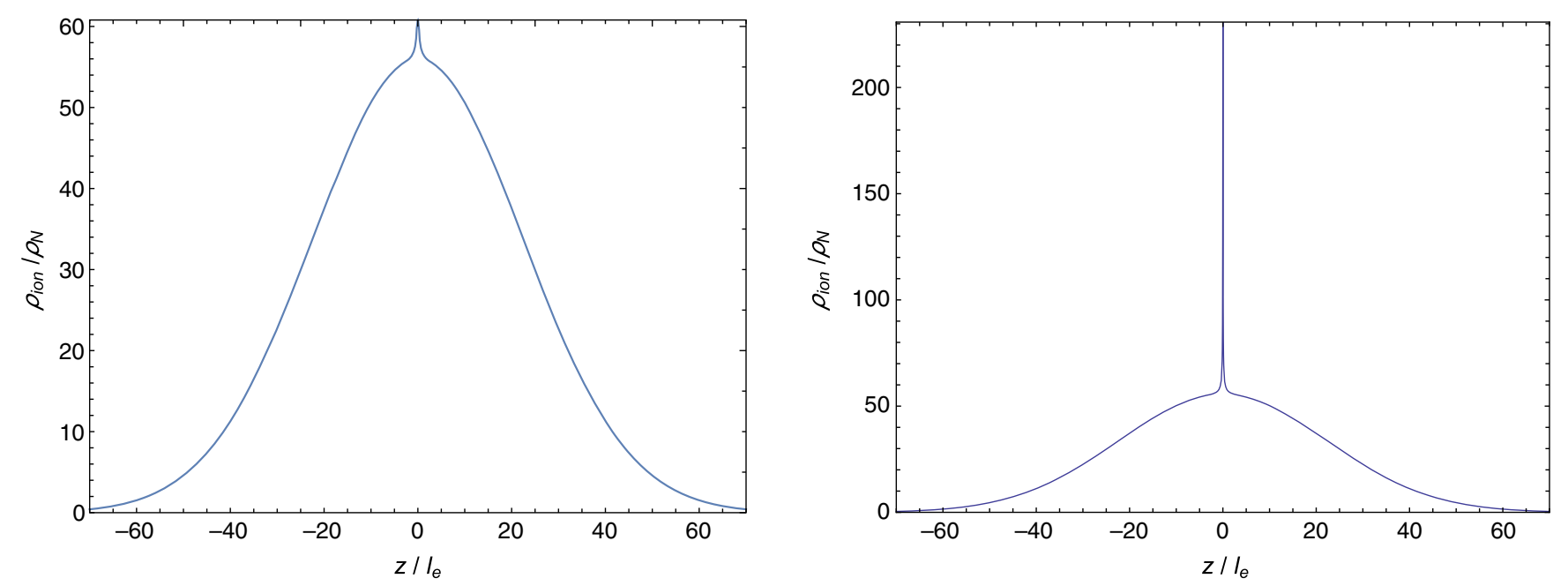

FIG. 1. Ion beam line density profile, $\rho_{\text {ion }}(t, z)$, as calculated from Eq. (26). The abscissa is the longitudinal distance from the center of electron bunch in units of one-half the electron full bunch length, $l_{e}$. The ordinate is the line density of the ion bunch in units of $\rho_{N}$. (Left) Ion beam profile after $t=2 \zeta_{0}^{-1}$. (Right) Ion beam profile after $t=10 \zeta_{0}^{-1}$. For both plots, it is assumed that $I_{e} / I_{\text {ion }}=10^{-3}$.

$$
h(I)=\frac{1}{\zeta_{0}} \int \frac{1+\frac{I}{I_{e}}}{I} d I=\frac{1}{\zeta_{0}} \ln \left[\frac{I}{I_{e}} \exp \left(\frac{I}{I_{e}}\right)\right],
$$

and the inverse function of $h(I)$ is given by solving for $I$ in the equation

$$
h(I)=\frac{1}{\zeta_{0}} \ln \left[\frac{I}{I_{e}} \exp \left(\frac{I}{I_{e}}\right)\right]=C
$$

The solution of Eq. (18) reads

$$
I=h^{-1}(C)=I_{e} P_{\log }\left[\exp \left(\zeta_{0} C\right)\right]
$$

where the product logarithm function, $P_{\log }(x)=w^{-1}(x)$, is the inverse function of

$$
w(x)=x e^{x} .
$$

Inserting Eq. (19) into Eq. (13) generates

$F(I, t)=\left(1+\frac{I_{e}}{I}\right) \cdot \frac{P_{\log }\left[\exp \left(\zeta_{0} C\right)\right] F_{0}\left\{I_{e} P_{\log }\left[\exp \left(\zeta_{0} C\right)\right]\right\}}{1+P_{\log }\left[\exp \left(\zeta_{0} C\right)\right]}$.

We take the initial ion distribution as

$$
F_{0}(I)=\frac{N}{2 \pi I_{\text {ion }}} \exp \left(-\frac{I}{I_{\text {ion }}}\right)
$$

where $I_{\text {ion }}$ is a parameter determined by the longitudinal emittance of the ion bunch. Inserting Eqs. (15) and (17) into Eq. (14), and taking $t_{0}=0$ leads to

$$
C=t+\frac{1}{\zeta_{0}} \ln \left[\frac{I}{I_{e}} \exp \left(\frac{I}{I_{e}}\right)\right] .
$$

Making use of Eqs. (22) and (23), Eq. (21) becomes

$$
F(I, t)=\frac{N}{2 \pi I_{\text {ion }}} g\left(\frac{I}{I_{e}}\right),
$$

with

$$
\begin{aligned}
g(\eta, t)= & \left(1+\frac{1}{\eta}\right) \frac{P_{\log }\left[\eta \exp \left(\zeta_{0} t+\eta\right)\right]}{1+P_{\log }\left[\eta \exp \left(\zeta_{0} t+\eta\right)\right]} \\
& \times \exp \left(-\frac{I_{e}}{I_{\text {ion }}} P_{\log }\left[\eta \exp \left(\zeta_{0} t+\eta\right)\right]\right) .
\end{aligned}
$$

Using Eq. (8), the line number density profile of the ion beam is then given by the following expression:

$$
\begin{aligned}
\rho_{\text {ion }}(t, z) & =k_{r f} \int_{-\infty}^{\infty} F\left(\frac{1}{2} k_{r f}^{2} z^{2}+\frac{1}{2} P^{2}, t\right) d P \\
& =\rho_{N} \int_{-\infty}^{\infty} g\left(\frac{z^{2}}{l_{e}^{2}}+y^{2}, t\right) d y,
\end{aligned}
$$

where $z$ is the longitudinal location along the ion bunch, $l_{e}$ is one-half of electron full bunch length, $k_{r f}=2 \pi / \lambda_{r f}$ is the $\mathrm{rf}$ wave vector, $\lambda_{r f}$ is the $\mathrm{rf}$ wavelength, $\sigma_{i}$ is the rootmean-square (rms) bunch length of the ion beam, and

$$
\rho_{N} \equiv \frac{N}{2 \pi} \frac{l_{e}}{\sigma_{i}^{2}} .
$$

In deriving Eq. (26), we used

$$
l_{e} \equiv \frac{\phi_{e, \max }}{k_{r f}}=\frac{\sqrt{2 I_{e}}}{k_{r f}},
$$


with $\phi_{e, \max }$ as the maximal rf phase for an ion with the synchrotron oscillation action of $I_{e}$. From Eq. (22), we also obtained the initial rms bunch length of ion beam as

$$
\sigma_{i}=\frac{\sqrt{I_{\mathrm{ion}}}}{k_{r f}}=l_{e} \sqrt{\frac{I_{\mathrm{ion}}}{2 I_{e}}},
$$

and the initial rms energy spread of the ion beam is

$$
\sigma_{\delta}=\frac{\nu_{s}}{h|\eta|} \sqrt{I_{\text {ion }}}
$$

As an example of applying Eq. (26), Fig. 1 shows the line density of an ion bunch after being cooled by a short electron bunch sitting in its center with a cooling rate profile given by Eq. (16). The half-electron bunch length is $l_{e}=0.045 \sigma_{i}$. As shown in Fig. 1 (left), a small blip appears after the ion bunch is cooled for 2 times the local cooling time, $2 \zeta_{0}^{-1}$. After the cooling time increases to $10 \zeta_{0}^{-1}$, a dense core appears around the ion bunch center. Because the local blip appears much faster than the overall reduction of the longitudinal emittance, it could potentially be used as a diagnostic tool for optimizing the cooling of a $\mathrm{CeC}$ system. However, the formation of the core is both due to the localized cooling as well as the absence of a mechanism to drive the particles out of the dense region. As indicated in the next section, the core is smoothed out for a nonzero diffusion coefficient as the diffusive kicks tend to move the particle out of the dense region.

\section{NUMERICAL SOLUTIONS FOR FINITE DIFFUSION}

In the presence of a nonzero diffusion coefficient, finding the analytical solution of Eq. (10) is usually difficult; thus, a numerical approach is pursued. Using the following definitions of normalized variables, $\bar{D}(r) \equiv D\left(r^{2} I_{e}\right) / D(0)$, $\bar{\zeta}(r) \equiv \zeta\left(r^{2} I_{e}\right) / \zeta_{0}, \bar{D}_{0} \equiv D(0) /\left(\zeta_{0} I_{e}\right), \bar{t}=t \zeta_{0}, R(r, \bar{t}) \equiv$ $\frac{2 \pi I_{\text {ion }}}{N} F\left(r^{2} I_{e}, \bar{t} \zeta_{0}^{-1}\right)$, and

$$
r \equiv \sqrt{I / I_{e}},
$$

Eq. (1) can be rewritten as

$$
\begin{aligned}
& r \frac{\partial R(r, \bar{t})}{\partial \bar{t}}+\alpha(r) \frac{\partial R(r, \bar{t})}{\partial r}+\beta(r) \frac{\partial^{2} R(r, \bar{t})}{\partial r^{2}} \\
& \quad+\gamma(r) R(r, \bar{t})=0,
\end{aligned}
$$

where

$$
\begin{gathered}
\alpha(r)=-\frac{r^{2}}{2} \bar{\zeta}(r)-\frac{\bar{D}_{0}}{4} \bar{D}(r)-\frac{\bar{D}_{0} r}{4} \frac{d \bar{D}(r)}{d r}, \\
\beta(r)=-\frac{\bar{D}_{0} r}{4} \bar{D}(r),
\end{gathered}
$$

and

$$
\gamma(r)=-\frac{r^{2}}{2} \frac{d \bar{\zeta}(r)}{d r}-r \bar{\zeta}(r)
$$

The difference equation derived from Eq. (32) reads

$$
\begin{aligned}
& \frac{\beta_{j}}{\Delta r^{2}} R_{j-1}^{n+1}+\left(\frac{r_{j}}{\Delta \bar{t}}-\frac{\alpha_{j}}{\Delta r}-2 \frac{\beta_{j}}{\Delta r^{2}}+\gamma_{j}\right) R_{j}^{n+1} \\
& \quad+\left(\frac{\alpha_{j}}{\Delta r}+\frac{\beta_{j}}{\Delta r^{2}}\right) R_{j+1}^{n+1}=\frac{r_{j}}{\Delta \bar{t}} R_{j}^{n}
\end{aligned}
$$

for $2 \leq j<N$, with $N$ as the index of the last bin in the grid of $r$,

$$
-\frac{\alpha_{j}}{\Delta r} R_{1}^{n+1}+\frac{\alpha_{j}}{\Delta r} R_{2}^{n+1}=0
$$

for $j=1$, and

$\frac{\beta_{N}}{\Delta r^{2}} R_{N-1}^{n+1}+\left(\gamma_{N}+\frac{r_{N}}{\Delta t}-\frac{\alpha_{N}}{\Delta r}-2 \frac{\beta_{N}}{\Delta r^{2}}\right) R_{N}^{n+1}=\frac{r_{N}}{\Delta \bar{t}} R_{N}^{n}$

for $j=N$. In deriving Eqs. (37) and (38), the following boundary conditions are applied:

$$
\left.\frac{\partial R(r, \bar{t})}{\partial r}\right|_{r=0}=0,
$$

and

$$
\left.R(r, \bar{t})\right|_{r \rightarrow \infty}=0 .
$$

The numerical solution of Eqs. (36)-(38) is obtained by applying the subroutine, TRIDAG, from Numerical Recipes [13]. After obtaining the phase space density, $R(r, \bar{t})$, the line number density of the ion bunch is given by

$$
\rho(z, t)=\rho_{N} \int_{-\infty}^{\infty} R\left(\sqrt{y^{2}+\frac{z^{2}}{l_{e}^{2}}}, t \zeta_{0}\right) d y .
$$

Figure 2 shows the ion bunch line density profile after $3 \zeta_{0}^{-1}$ of cooling, where the initial ion distribution is given by Eq. (22), i.e.,

$$
R_{0}(r)=\exp \left(-r^{2} / r_{0}^{2}\right)
$$

with

$$
r_{0}^{2} \equiv I_{\mathrm{ion}} / I_{e} .
$$

To compare with the analytical result in Eq. (26), we take

$$
\bar{\zeta}(r)=\frac{1}{1+r^{2}} .
$$

The numerical method is applicable to the arbitrary dependence of the diffusion coefficient on the synchrotron 


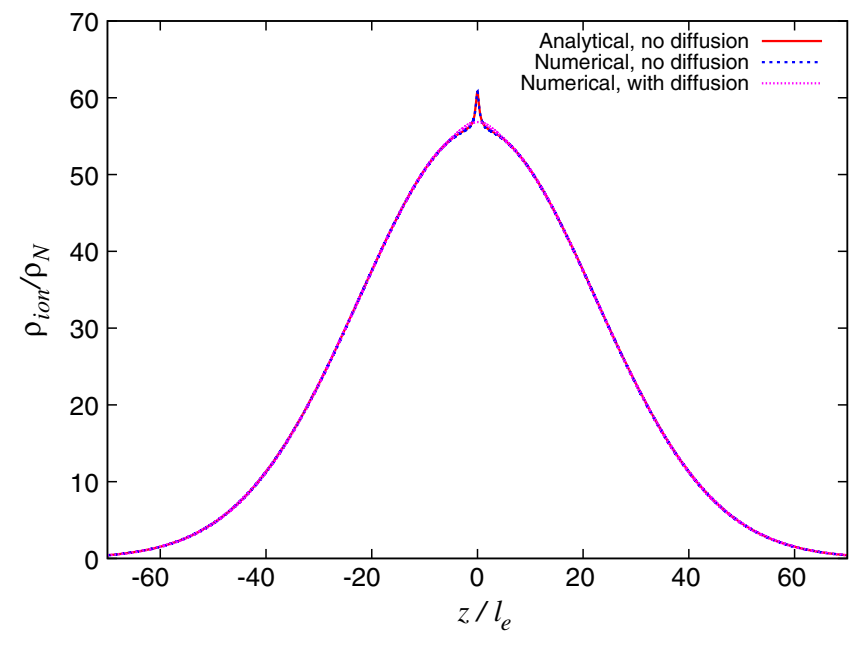

FIG. 2. Comparing the numerical solution of Eqs. (36)-(38) (blue) with the analytical solution calculated from Eq. (24) (red). The abscissa is the normalized longitudinal location in units of $l_{e}$, and the ordinate is the ion bunch line number density in units of $\rho_{N}$, as calculated from Eqs. (27) and (41). The red and blue curves show the results for zero diffusion. The magenta curve shows the numerical solution of Eqs. (36)-(38) with diffusion coefficient, $\bar{D}_{0}=100$, and the profile $\bar{D}(r)=1 /\left(1+r^{2}\right)$. All curves are plotted for $\bar{t}=2$ and $r_{0}^{2}=I_{\mathrm{ion}} / I_{e}=1000$.

oscillation amplitude. In Fig. 2 (magenta dots), to illustrate the effects of diffusion, we take

$$
D\left(r^{2} I_{e}\right)=\frac{\bar{D}_{0}}{1+r^{2}} I_{e} \zeta_{0}
$$

with $\bar{D}_{0}=100$ as an example. As shown in Fig. 2, the numerical solution (blue dashes) reproduces the analytical result (red solid) in the absence of diffusion. For the nonvanishing diffusion coefficient (magenta dots), however, the central blip is smoothed out. As indicated in the next section, the smoothing effects due to diffusion do not rely on a specific form for the cooling rate and diffusion coefficient, i.e., Eq. (44), and similar influences of diffusion to the ion bunch profile are observed for a more realistic cooling profile derived for the FEL-based $\mathrm{CeC}$ simulation. ${ }^{3}$

\section{EVALUATION FOR FEL-BASED COHERENT ELECTRON COOLING}

The assumed dependence of the cooling rate on the synchrotron oscillation amplitude in Eq. (16) is convenient for obtaining an analytical solution, Eq. (24), as well as

\footnotetext{
${ }^{3}$ A qualitative estimate of whether a local blip of certain length scale is possible to form can be made by using Eq. (9). For $\zeta$ and $D$ independent of $I$, Eq. (9) reduces to $F_{\text {eq }}(I)=A e^{-I / I_{b}}$ with $I_{b}=D / \zeta$. Thus, around the center of ion bunch $(r \approx 0)$, Eq. (45) suggests that the local blip cannot have a length scale much smaller than $z_{b} \approx l_{e} \sqrt{\bar{D}_{0}}$.
}

validating the numerical method for solving Eqs. (36)-(38). In practice, however, the dependences of both the cooling rate and diffusion coefficient on the synchrotron amplitude, after averaging over one synchrotron oscillation period, should be determined by the specific cooling scheme, such as the longitudinal profile of the electron bunch, whether the electron bunch is painted around the ion bunch, and how the cooling force and diffusive kick depend on local electron properties. The single pass energy kick received by an ion as it travels through the storage ring is given by

$$
\begin{aligned}
\Delta \delta \gamma_{j, n}= & \delta \gamma_{j, n+1}-\delta \gamma_{j, n}=-g_{\gamma} \sin \left(\frac{2 \pi R_{56}}{\lambda_{0} \gamma_{0}} \delta \gamma_{j, n}\right) \\
& +d_{\mathrm{ion}} X_{j, n}+d_{e} Y_{j, n}+d_{\mathrm{IBS}} Z_{j, n}
\end{aligned}
$$

where

$$
\delta \gamma_{j, n}=\frac{\nu_{s} \gamma_{0}}{h|\eta|} P_{j, n}
$$

is the energy deviation (normalized to its rest energy, $A_{i} m_{u} c^{2}$ ) of the $j$ th ion in its $n$th circulation around the ring,

$$
g_{\gamma} \equiv \frac{Z_{i} e E_{p} l}{A_{i} m_{u} c^{2}}
$$

is the amplitude of the self-induced coherent energy kick received by the ion in the cooling section, $\lambda_{0}$ is the optical wavelength of the FEL amplifier, $f_{\text {rev }}$ is the revolution frequency of the ions, and $R_{56}$ is the longitudinal dispersion from the modulator to the kicker of the cooling section. Equation (46) suggests that for ions with large energy deviation, $\left|\delta \gamma_{j}\right|>\frac{\lambda_{0} \gamma_{0}}{2 R_{56}}$, the coherent kick may lead to anticooling. In practice, to avoid anticooling, the ion beam should be conditioned such that its rms energy spread is much smaller than $\frac{\lambda_{0} \gamma_{0}}{2 R_{56}}$. In addition, for ions with $\left|\delta \gamma_{j}\right| \ll$ $\frac{\lambda_{0} \gamma_{0}}{2 \pi R_{56}}$, Eq. (46) can be approximated as

$\Delta \delta \gamma_{j, n}=-g_{\gamma} \frac{2 \pi R_{56}}{\lambda_{0} \gamma_{0}} \delta \gamma_{j, n}+d_{\mathrm{ion}} X_{j, n}+d_{e} Y_{j, n}+d_{\mathrm{IBS}} Z_{j, n}$.

For simplicity, we assume the relative energy spread of the ions satisfes

$$
\sigma_{\delta} \ll \frac{\lambda_{0}}{2 \pi R_{56}}
$$

and use the linear cooling force, Eq. (49), to derive the cooling rate and diffusion coefficients in Eq. (1). As we will see in Sec. VI, overestimates of cooling may occur if Eq. (50) is not strictly satisfied. The local cooling time, $T_{0}$, and its inversion, the local cooling rate, $\zeta_{0}$, are related to the coherent energy kick, $g_{\gamma}$, by 

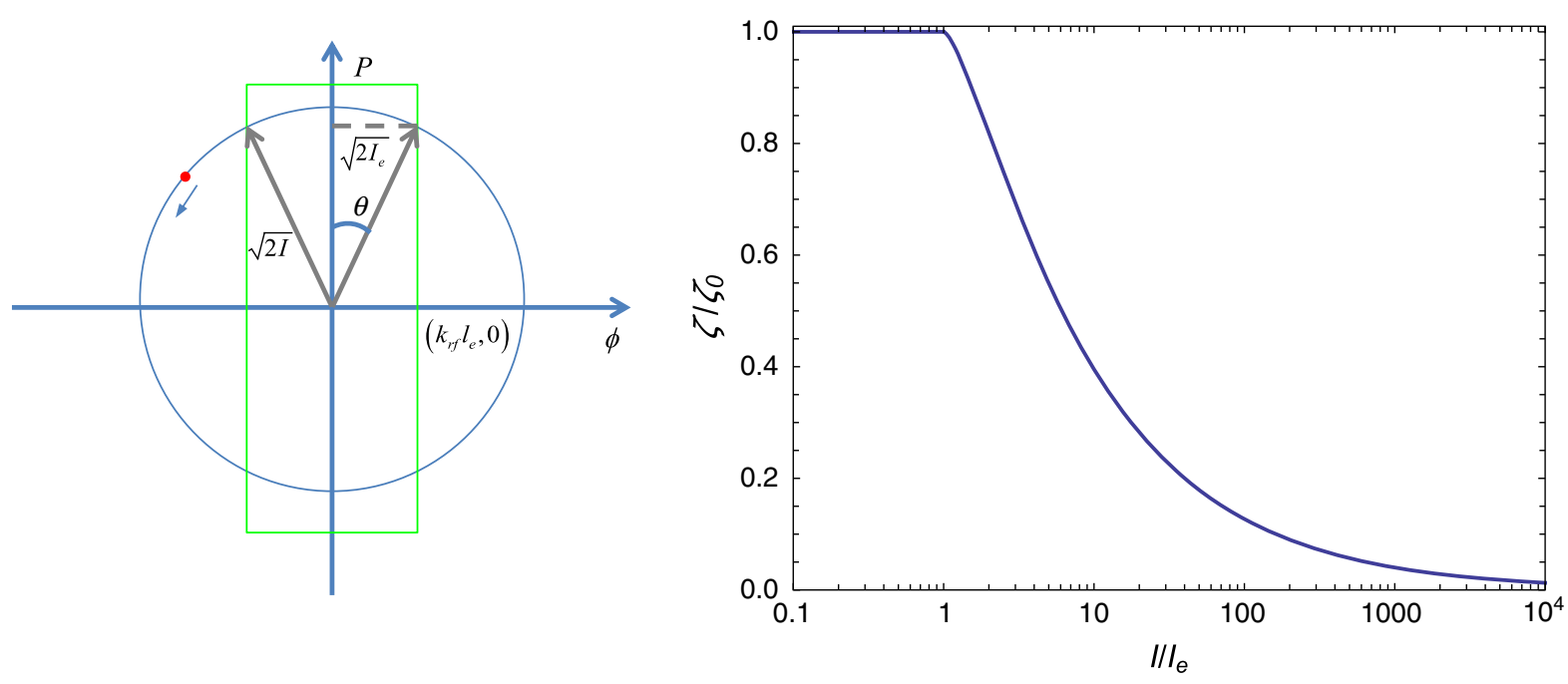

FIG. 3. Averaged cooling rate over a synchrotron oscillation period. (Left) Illustration of an ion passing through the cooling electron beam while conducting synchrotron oscillation in its longitudinal phase space, with the green box representing the longitudinal range of the electron bunch and the red dot representing the ion. (Right) Averaged cooling rate as calculated from Eq. (56) in units of $\zeta_{0}$ [see Eq. (51)]. The abscissa in the right plot is the action of the ion's synchrotron oscillation in units of $I_{e}=k_{r f}^{2} l_{e}^{2} / 2$.

$$
T_{0}=\zeta_{0}^{-1}=\frac{1}{f_{\text {rev }}} \cdot \frac{\lambda_{0} \gamma_{0}}{g_{\gamma} 2 \pi R_{56}} .
$$

For each circulation around the ring, the reduction in the ion's longitudinal oscillation action due to cooling is given by

$$
\Delta I_{c}=\frac{1}{2} \Delta\left(P^{2}+\phi^{2}\right)=P \Delta P_{c},
$$

where

$$
\Delta P_{c}=\frac{h|\eta|}{\nu_{s} \gamma} \Delta \delta \gamma_{c}
$$

Inserting

$$
\Delta \delta \gamma_{c}=-g_{\gamma} \frac{2 \pi R_{56}}{\lambda_{0} \gamma_{0}} \delta \gamma=-\zeta_{0} T_{\text {rev }} \delta \gamma
$$

into Eq. (52) and using Eq. (47) gives

$$
\Delta I_{c}=-\zeta_{0} T_{\mathrm{rev}} P^{2}=-2 I \zeta_{0} T_{\mathrm{rev}} \cos ^{2} w,
$$

where $T_{\text {rev }}$ is the revolution period of the ion, and we use Eq. (2) in deriving the second equation of (55). Averaging Eq. (55) over one synchrotron oscillation leads to

$$
\zeta(r)=-\frac{1}{I}\left\langle\frac{\Delta I_{c}}{T_{\text {rev }}}\right\rangle_{T_{s}}=\zeta_{0} \bar{\zeta}(r)
$$

where $r=\sqrt{I / I_{e}}, I_{e}=\frac{1}{2}\left(k_{r f} l_{e}\right)^{2}$, and $^{4}$

\footnotetext{
${ }^{4}$ It is worth noting that the second term in Eq. (57) for $r \geq 1$ is related to the fact that ions tend to have large energy deviation when they are close to the bunch center and overlap with cooling electrons, which makes them cooled more efficiently.
}

$$
\bar{\zeta}(r)= \begin{cases}\frac{2}{\pi} \arcsin \left(\frac{1}{r}\right)+\frac{2}{\pi r^{2}} \sqrt{r^{2}-1} ; & \text { for } r \geq 1 \\ 1 ; & \text { for } r<1\end{cases}
$$

and for $r>1$, we used the following relation to derive Eq. (57):

$$
\left\langle\cos ^{2} w\right\rangle_{T_{s}}=\frac{2}{2 \pi} \int_{-\theta}^{\theta} \cos ^{2} w d w=\frac{\theta}{\pi}+\frac{1}{\pi} \sin \theta \cos \theta,
$$

with $\sin \theta=\sqrt{I_{e} / I}=1 / r$ as shown in Fig. $3^{5}$ (left). Figure 3 (right) shows how the cooling rate depends on the synchrotron oscillation action of the particle, as calculated from Eq. (57).

The second and the third term in the rhs of Eq. (46) represent the diffusive kicks received by the ion in the cooling section (Appendix B), where $X_{j, n}$ and $Y_{j, n}$ are random numbers uniformly distributed from -1 to 1 ,

$d_{\text {ion }}=g_{\gamma} \sqrt{\frac{3}{2} \sqrt{\pi} \rho_{\text {ion }}\left(z_{j}\right) \sigma_{w}} \approx g_{\gamma} \sqrt{\frac{3}{2} \sqrt{\pi} \rho_{\text {ion }}\left(z_{e}\right) \sigma_{w}}$

is the amplitude of the incoherent energy kick induced by all neighboring ions,

\footnotetext{
${ }^{5}$ For an ion with maximal energy deviation of $\sigma_{\delta}=\frac{\nu_{s} k_{r f}}{h|\eta|} \sigma_{i}$, its horizontal coordinate in the right plot is given by Eq. (29), or explicitly $I / I_{e}=r^{2}=2 \sigma_{i}^{2} / l_{e}^{2}$. For example, with the parameters in Table I, an ion with energy deviation of $\sigma_{\delta}=3.35 \times 10^{-4}$ resides at $r=346$ or $I / I_{e}=1.2 \times 10^{5}$.
} 


$$
d_{e}=\frac{g_{\gamma}}{Z_{i}} \sqrt{\frac{3}{2} \sqrt{\pi} \rho_{e}\left(z_{j}\right) \sigma_{w}} \approx \frac{g_{\gamma}}{Z_{i}} \sqrt{\frac{3}{2} \sqrt{\pi} \rho_{e} \sigma_{w}}
$$

is the amplitude of the incoherent energy kick induced by all neighboring electrons, and $\sigma_{w}$ is the coherent length of the density wave packet induced by the ion in the electron beam. In deriving the second equation of Eqs. (59) and (60), we assume that the center of the electron bunch is located at $z_{e}$, the ion beam line density does not vary significantly over the range of the cooling electron beam, and the electron beam has uniform line density. The last term of the rhs of Eq. (46) is responsible for the accumulated energy kicks due to intrabeam scattering (IBS) while ions travel through the ring. $Z_{j, n}$ is a random number uniformly distributed from -1 to 1 and the kick strength, $d_{\mathrm{IBS}}$, is to be determined by the IBS growth rate, as obtained from the Piwinski formulas [14,15] (Appendix C).

For an ion with synchrotron oscillation action, $I$, a random energy kick in its $i$ th circulation, $d \cdot X_{i}$, corresponds to a change in its longitudinal action of

$$
\Delta I_{d}=P \Delta P_{d}=h \frac{|\eta|}{\nu_{s}} \frac{d}{\gamma_{0}} X \sqrt{2 I} \cos w .
$$

After averaging over random variable, $X$, and synchrotron phase, $w$, the rms variation of $\Delta I$ is

$$
\left\langle\Delta I_{d}^{2}\right\rangle=\frac{1}{3}\left(h \frac{|\eta|}{\nu_{s}}\right)^{2}\left(\frac{d}{\gamma_{0}}\right)^{2} 2 I\left\langle\cos ^{2} w\right\rangle_{T_{s}} .
$$

Using Eq. (58), the diffusion coefficient in the FokkerPlanck equation, Eq. (1), is given by [16]

$$
D(I)=\frac{\left\langle\Delta I_{d}^{2}\right\rangle}{2 T_{\mathrm{rev}} I}=D(0) \bar{D}(I)
$$

with

$$
D(0)=\frac{d^{2}}{6 T_{\text {rev }} \gamma^{2}}\left(h \frac{|\eta|}{\nu_{s}}\right)^{2} .
$$

The normalized diffusion coefficient, $\bar{D}_{0} \equiv D(0) /$ $\left(I_{e} \zeta_{0}\right)$, is

$$
\bar{D}_{0}=\frac{d^{2}}{6 T_{\mathrm{rev}} \zeta_{0} I_{e} \gamma^{2}}\left(h \frac{|\eta|}{\nu_{s}}\right)^{2}=\frac{d^{2}}{3 T_{\mathrm{rev}} \zeta_{0}}\left(\frac{\sigma_{i}}{l_{e} \gamma \sigma_{\delta}}\right)^{2},
$$

where in deriving Eq. (65), we used Eqs. (29) and (30), i.e.,

$$
I_{e}=\frac{l_{e}^{2}}{2 \sigma_{i}^{2}} I_{\mathrm{ion}}=\frac{l_{e}^{2}}{2 \sigma_{i}^{2}}\left(h \frac{|\eta|}{\nu_{s}} \sigma_{\delta}\right)^{2} .
$$

Combining Eqs. (58), (62), (63), and (65), we obtain the diffusion coefficient for the incoherent kicks along the cooling section

$$
D_{\text {cec }}(r)=\zeta_{0} I_{e} \bar{D}_{0, \operatorname{cec}} \bar{D}_{\text {cec }}(r)
$$

with

$$
\bar{D}_{0, \mathrm{cec}}=\frac{d_{e}^{2}+d_{\mathrm{ion}}^{2}}{3 T_{\mathrm{rev}} \zeta_{0}}\left(\frac{\sigma_{i}}{l_{e} \gamma \sigma_{\delta}}\right)^{2}
$$

and

$$
\bar{D}(r)= \begin{cases}\frac{2}{\pi} \arcsin \left(\frac{1}{r}\right)+\frac{2}{\pi r^{2}} \sqrt{r^{2}-1} ; & \text { for } r \geq 1 \\ 1 ; & \text { for } r<1 .\end{cases}
$$

For the next step, we calculate the diffusion coefficient due to IBS. Similar to Eq. (61), the increase in the longitudinal action due to the IBS kick is

$$
\Delta I_{\mathrm{IBS}}=P \Delta P_{\mathrm{IBS}}=h \frac{|\eta|}{\nu_{s}} \frac{d_{\mathrm{IBS}}(\phi)}{\gamma_{0}} X \sqrt{2 I} \cos w,
$$

where $d_{\mathrm{IBS}}(\phi)$ is the IBS kick strength, which is proportional to the square root of the local ion density. Making use of Eqs. (22) and (29), we obtain

$$
\begin{aligned}
d_{\mathrm{IBS}}(\phi) & =d_{0, \mathrm{IBS}} \exp \left(-\frac{\phi^{2}}{4 k_{r f}^{2} \sigma_{i}^{2}}\right) \\
& =d_{0, \mathrm{IBS}} \exp \left(\frac{-I}{2 I_{\mathrm{ion}}} \sin ^{2} w\right),
\end{aligned}
$$

with $d_{0, \text { IBS }}$ as the kick strength at the bunch center. The variance of the action kick is obtained from Eq. (70)

$$
\begin{aligned}
\left\langle\Delta I_{\mathrm{IBS}}^{2}\right\rangle= & \frac{2 I d_{0, \mathrm{IBS}}^{2}}{3}\left(h \frac{|\eta|}{\nu_{s}}\right)^{2}\left(\frac{1}{\gamma_{0}}\right)^{2} \\
& \times\left\langle\exp \left(\frac{-I}{I_{\text {ion }}} \sin ^{2} w\right) \cos ^{2} w\right\rangle_{T_{s}} .
\end{aligned}
$$

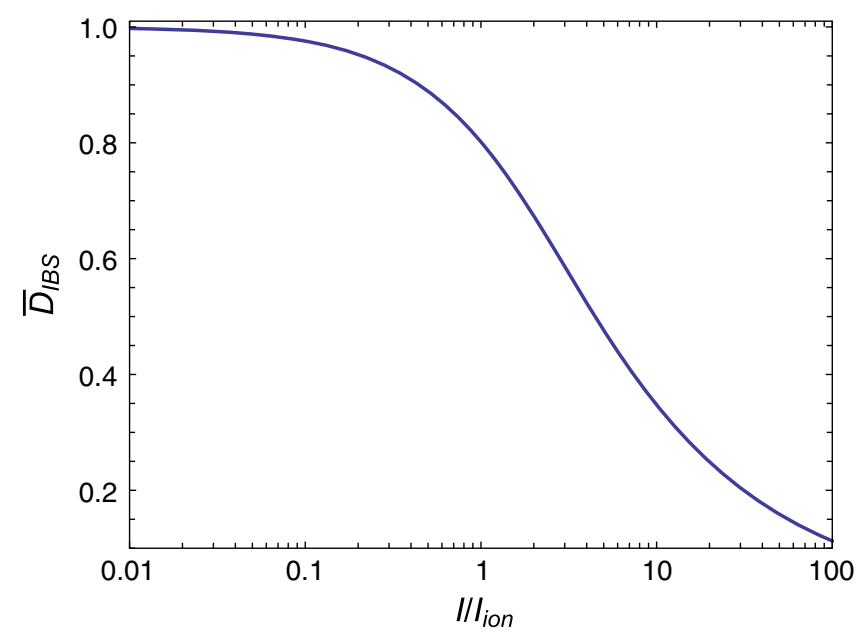

FIG. 4. Dependence of IBS-induced diffusion coefficient on the synchrotron oscillation action of an ion. The ordinate is the normalized diffusion coefficient induced by IBS as calculated from Eq. (76), and the abscissa is the synchrotron oscillation action of an ion in units of $I_{\text {ion }}=k_{r f}^{2} \sigma_{i}^{2}$. 
TABLE I. Beam parameters for the proof of the $\mathrm{CeC}$ principle experiment.

Electron beam parameters

Peak current, A

Full bunch length, ps

Norm. emittance, rms, $\mu \mathrm{m}$

Relative energy spread, rms

Beam energy, $\gamma$

rms beam width at modulator and kicker, mm

rms beam width at FEL amplifier, mm
Ion beam parameters

$\begin{array}{cc}\text { Charge number, } Z_{\text {ion }} & 79 \\ \text { Bunch intensity } & 10^{8} \\ \text { Bunch length, rms, ns } & 3.06 \\ \text { Relative energy spread, rms } & 3.35 \times 10^{-4} \\ \text { rf frequency, MHz } & 28 \\ \text { width at modulator and kicker, mm } & 0.7 \\ \text { width at modulator and kicker, mm } & 0.7\end{array}$

TABLE II. System parameters of the proof of the CeC principle experiment.

\begin{tabular}{lccc}
\hline \hline Gain of FEL amplification & 80 & FEL wavelength, $\mu \mathrm{m}$ & 30.5 \\
FEL parameter, $a_{w}$ & 0.5 & FEL length, $\mathrm{m}$ & 7.5 \\
FEL type & Helical & Peak correcting field, V/m & 36 \\
Kicker length, $\mathrm{m}$ & 3 & $R_{56}, \mathrm{~cm}$ & 1.2 \\
Coherent kick amplitude, $g_{\gamma}$ & $4.657 \times 10^{-8}$ & Coherent length, $\sigma_{w}, \mathrm{~mm}$ & 0.54 \\
CeC diffusive kick from neighboring ions, $d_{\text {ion }}$ & $1.163 \times 10^{-5}$ & Local cooling time $\left(T_{0}\right), \mathrm{s}$ & 3.185 \\
IBS diffusive kick at bunch center, $d_{\mathrm{IBS}}(0)$ & $1.886 \times 10^{-6}$ & CeC diffusive kick from electrons, $d_{e}$ & $2.038 \times 10^{-5}$ \\
\hline \hline
\end{tabular}

With help from Eq. (63) and the following relation,

$$
\begin{aligned}
\left\langle\exp \left(-z \sin ^{2} w\right) \cos ^{2} w\right\rangle_{T_{s}} & =\frac{1}{2 \pi} \int_{0}^{2 \pi} \exp \left(-z \sin ^{2} w\right) \cos ^{2} w d w \\
& =\frac{1}{2} e^{-\frac{z}{2}}\left[I_{0}\left(\frac{z}{2}\right)+I_{1}\left(\frac{z}{2}\right)\right], \quad
\end{aligned}
$$

we obtain

$$
D_{\mathrm{IBS}}(I)=\frac{\left\langle\Delta I_{\mathrm{IBS}}^{2}\right\rangle}{2 T_{\mathrm{rev}} I}=I_{e} \zeta_{0} \bar{D}_{0, \mathrm{IBS}} \bar{D}_{\mathrm{IBS}}(I),
$$

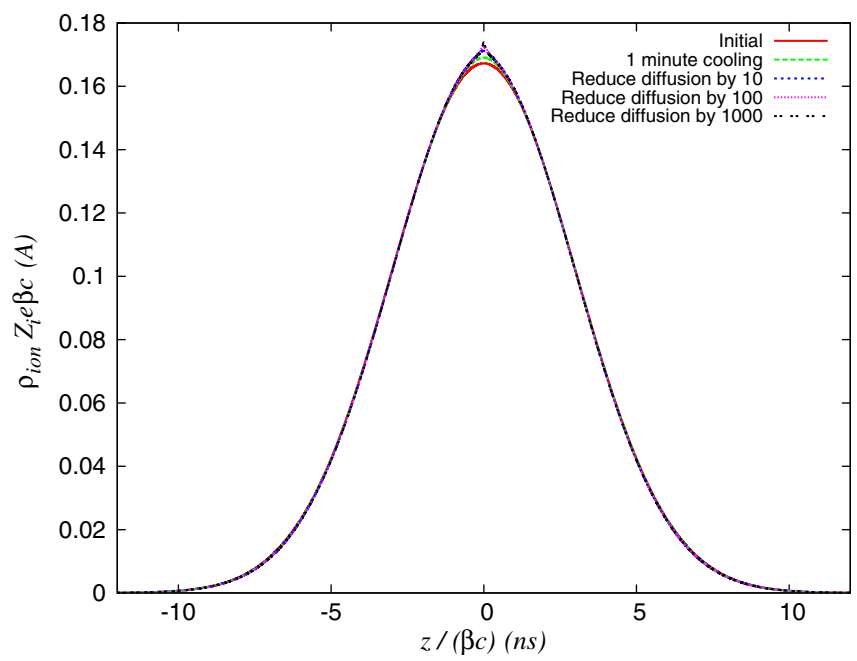

where $I_{0}(x)$ and $I_{1}(x)$ are modified Bessel functions,

$$
\bar{D}_{0, \mathrm{IBS}}=\frac{d_{0, \mathrm{IBS}}^{2}}{3 T_{\mathrm{rev}} \zeta_{0}}\left(\frac{\sigma_{i}}{l_{e} \gamma \sigma_{\delta}}\right)^{2},
$$

and

$$
\bar{D}_{\mathrm{IBS}}(I)=e^{-\frac{I}{2 I_{\text {ion }}}}\left[I_{0}\left(\frac{I}{2 I_{\text {ion }}}\right)+I_{1}\left(\frac{I}{2 I_{\text {ion }}}\right)\right] .
$$

Fig. 4 shows how the diffusion coefficient induced by IBS depends on the synchrotron oscillation action of an ion,

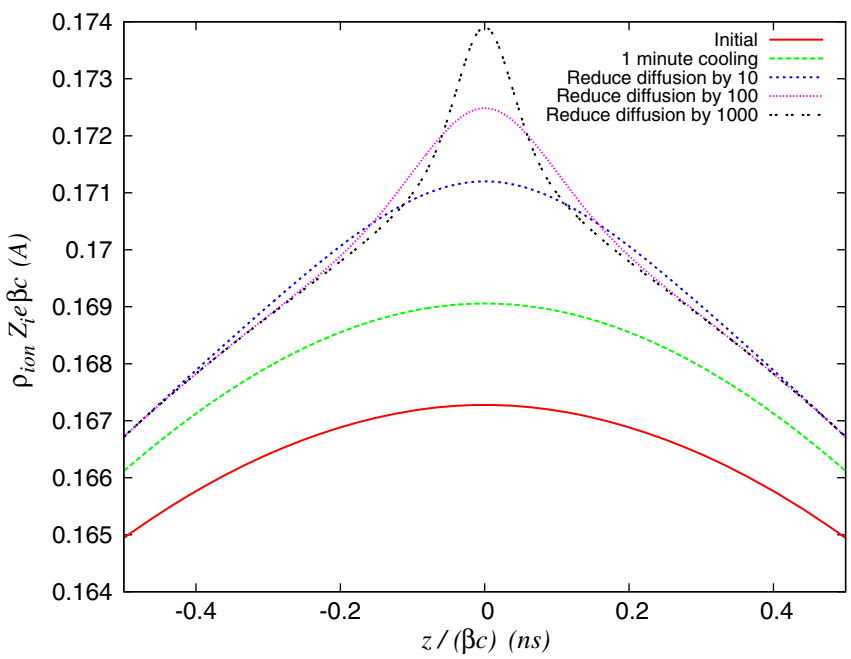

FIG. 5. Ion bunch current profile after $1 \mathrm{~min}$ of cooling and its dependence on the diffusion coefficient. These plots are generated by solving Eqs. (36)-(38) with the parameters listed in Tables I and II. The cooling rate and diffusion coefficients are calculated from Eqs. (56) and (79), respectively. The red curve shows the initial ion bunch current profile, and the green curve shows the ion bunch profile after 1 min of cooling. The blue, magenta, and black curves show the ion bunch profiles after 1 min of cooling with the diffusion coefficient reduced by factors of 10, 100, and 1000 from the nominal values (green). The left plot shows the overall bunch profile, and the right plot shows the zoomed-in region around the bunch center. 


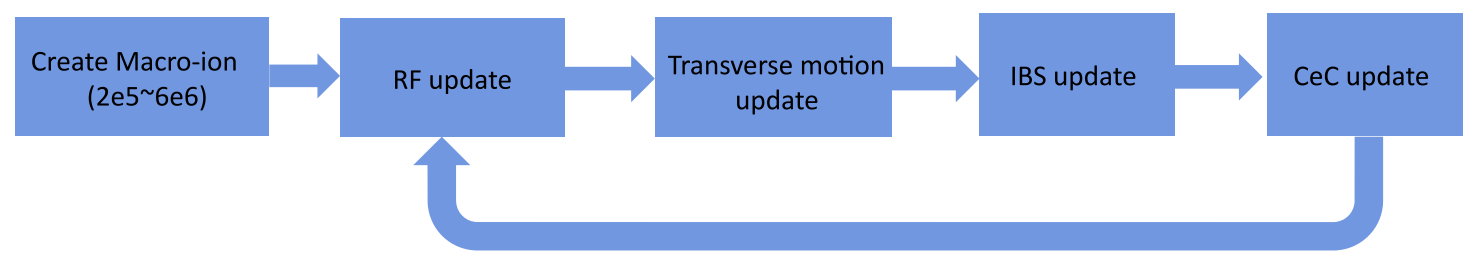

FIG. 6. Flowchart illustration of the tracking process.

as calculated from Eq. (76). Inserting Eqs. (31) and (43) into Eq. (74) yields

$$
D_{\mathrm{IBS}}(r)=I_{e} \zeta_{0} \bar{D}_{0, \mathrm{IBS}} \bar{D}_{\mathrm{IBS}}(r),
$$

with

$$
\bar{D}_{\mathrm{IBS}}(r)=e^{-\frac{r^{2}}{2 r_{0}^{2}}}\left[I_{0}\left(\frac{r^{2}}{2 r_{0}^{2}}\right)+I_{1}\left(\frac{r^{2}}{2 r_{0}^{2}}\right)\right]
$$

The total diffusion coefficient is obtained by the summation of Eqs. (67) and (77):

$$
D(r)=D_{\text {cec }}(r)+D_{\mathrm{IBS}}(r) .
$$

For the parameters of the proof of the $\mathrm{CeC}$ principle experiment listed in Tables I and II ${ }^{6,7}$ [17], the normalized diffusion coefficients are $\bar{D}_{0, \text { cec }}=2.97 \times 10^{4}$ and $\bar{D}_{0, \mathrm{IBS}}=$ 192, respectively.

Figure 5 (right) shows the ion bunch current profile around the bunch center after $\sim 1$ min of cooling with the nominal (green) and artificially reduced diffusion coefficients (blue, magenta, and black). As shown in Fig. 5 (right), the local blip is fully suppressed for the nominal parameters and starts to be visible if the normalized diffusion coefficient is reduced by 2 orders of magnitudes or more.

According to Eqs. (59), (60), and (68), the normalized diffusion coefficients due to $\mathrm{CeC}, \bar{D}_{0, \text { cec }}$, decreases linearly with $g_{\gamma}$. One way to reduce diffusion in the $\mathrm{CeC}$ process is to reduce the self-induced energy kick, $g_{\gamma}$. However, local cooling time, $T_{0}$, increases proportionally with $g_{\gamma}^{-1}$; hence, the normalized maximal diffusion coefficient due to IBS, $\bar{D}_{0, \text { IBS }}$, increases linearly with $g_{\gamma}^{-1}$. In addition, increasing $T_{0}$ will also slow the overall process, defeating the purpose of using the blip as a fast-diagnostic signal.

\footnotetext{
${ }^{6}$ The gain of the FEL amplifier is around the optimal value for the parameters in Tables I and II. Theoretical calculation of the optimal gain can be found from [3,9].

${ }^{7}$ These parameters were planned for the FEL-based $\mathrm{CeC}$ experiment at the time when these calculations were performed. They are subject to change as the understandings of the $\mathrm{CeC}$ processes develop.
}

\section{MACROPARTICLE TRACKING}

The numerical method for solving the Fokker-Planck equation as developed in Sec. IV has the advantages of necessitating minimal computational time (few minutes on a pc) and resources. However, its applicability is limited to the scheme in which the cooling rate and diffusion coefficient do not change significantly throughout the process, the ion bunch length is small enough that the Hamiltonian of Eq. (15) is accurate, and the cooling force is proportional to the energy deviation of the particle. To cross-check the results obtained in Sec. V as well as provide a more versatile tool for a general cooling scheme, we have developed a macroparticle tracking code. As shown in Fig. 6, typically 0.2-6 millions of macro-ions are generated when the simulation starts. The longitudinal coordinates of each macro-ion are then updated according to the rf voltage experienced and the phase slip factor of the lattice:

$$
\bar{\varepsilon}=\varepsilon+\frac{q}{m c^{2}} V_{r f}(\tau),
$$

and

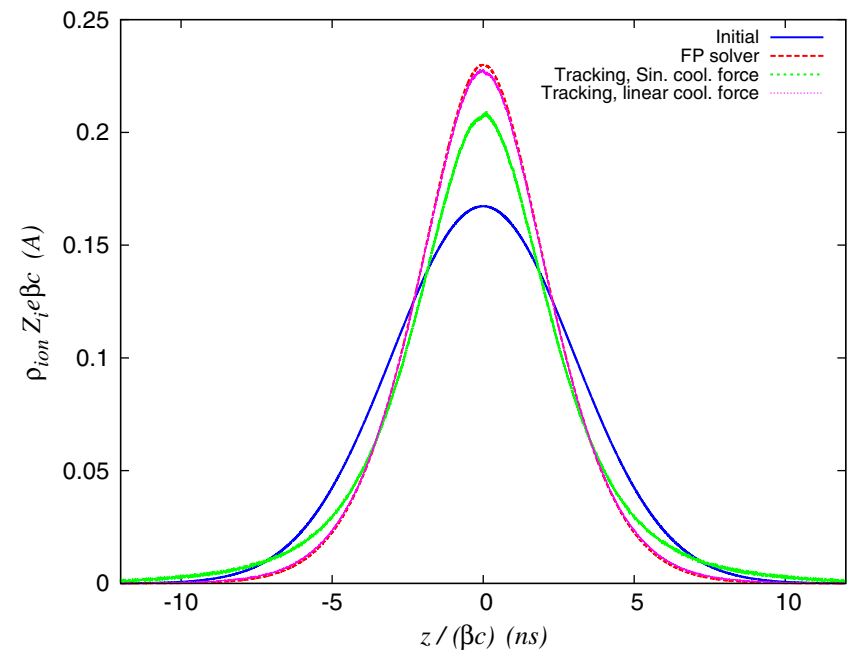

FIG. 7. The ion bunch current profiles after $40 \mathrm{~min}$ of cooling as obtained by solving Eqs. (36)-(38) (red) and through macroparticle tracking (green and magenta). The green curve shows the tracking results with the sinusoidal cooling force, as described by Eq. (46), while the magenta curve shows the tracking results with the linear cooling force, given by Eq. (49). 


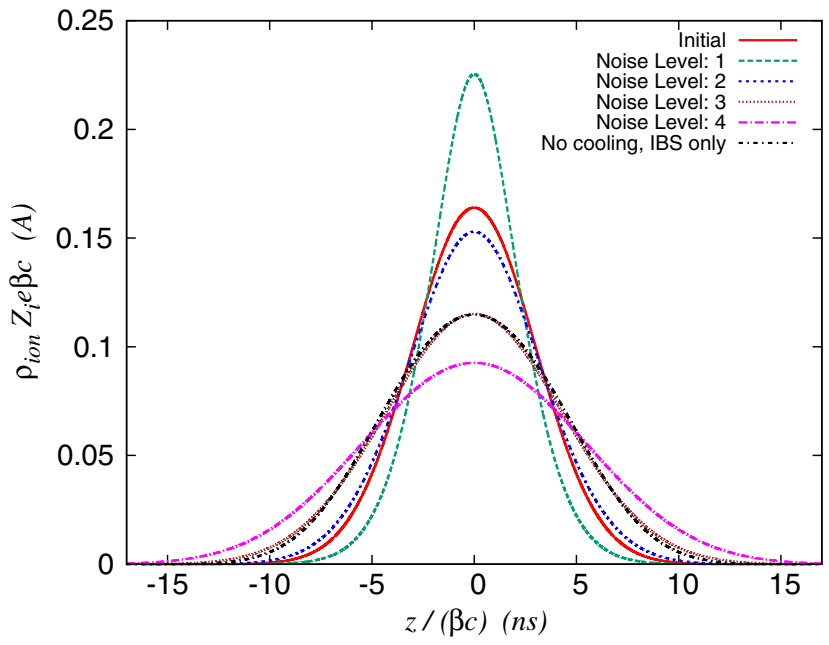

(a)

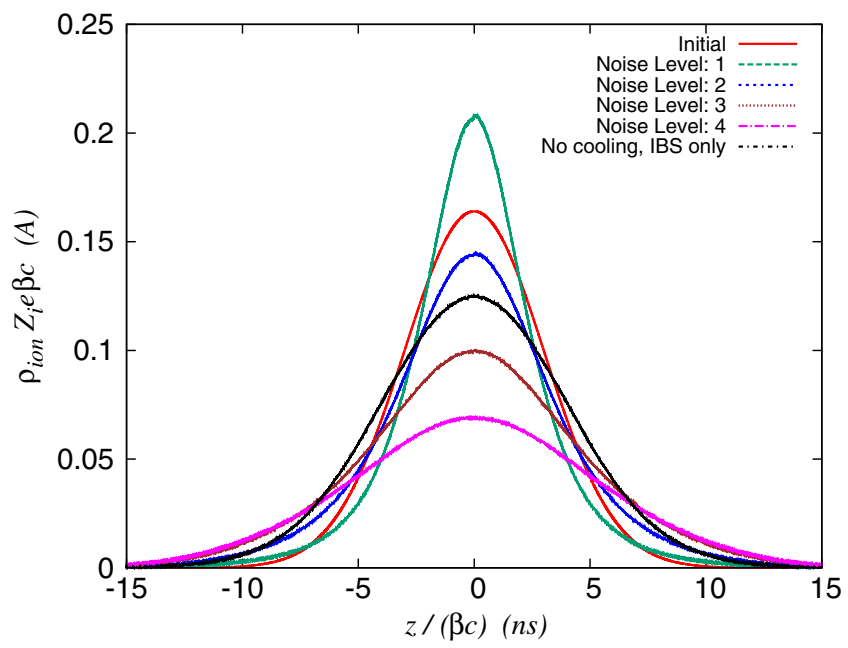

(b)

FIG. 8. Tolerance of the proof of the $\mathrm{CeC}$ principle experiment on the noise level in the electrons. (a) The plots are obtained by solving Eqs. (36)-(38); (b) the plots are obtained by macroparticle tracking. The red dashed curve is the initial ion bunch current profile, and the black curve is the current profile of a witness bunch, i.e., an ion bunch not overlapping with electron bunch, after 40 min of storage. The green curve is the ion bunch profile after $40 \mathrm{~min}$ of cooling by electrons with a natural noise level $\left(d_{e}=2.038 \times 10^{-5}\right.$ from Table I is used in the calculation). To account for the excessive noise in the electrons, increased values of $d_{e}$ are used in generating blue $\left(d_{e}=4.076 \times 10^{-5}\right)$, brown $\left(d_{e}=6.114 \times 10^{-5}\right)$, and magenta $\left(d_{e}=8.152 \times 10^{-5}\right)$ curves.

$$
\bar{\tau}=\tau+\frac{T_{\text {rev }} \eta}{\beta^{2} \gamma_{0}} \bar{\varepsilon},
$$

where $q$ is the charge of the ion, $m$ is the mass of the ion, $c$ is speed of light, $\varepsilon$ is the energy deviation of the macro-ion in units of $m c^{2}, \tau$ is the arriving time of the macro-ion, $V_{r f}(\tau)$ is the rf voltage experienced by the ion, $\gamma_{0} m c^{2}$ is the energy of the reference ion, $T_{\text {rev }}$ is the revolution period, and $\eta$ is the phase slip factor. The update of the transverse coordinate uses a one-turn linear transfer matrix:

$$
\left(\begin{array}{c}
\bar{x} \\
\bar{p}_{x} \\
\bar{y} \\
\bar{p}_{y}
\end{array}\right)=\left(\begin{array}{cccc}
\cos \psi_{x} & \sin \psi_{x} & 0 & 0 \\
-\sin \psi_{x} & \cos \psi_{x} & 0 & 0 \\
0 & 0 & \cos \psi_{y} & \sin \psi_{y} \\
0 & 0 & -\sin \psi_{y} & \cos \psi_{y}
\end{array}\right)\left(\begin{array}{c}
\bar{x} \\
\bar{p}_{x} \\
\bar{y} \\
\bar{p}_{y}
\end{array}\right),
$$

where $\psi_{x}$ and $\psi_{y}$ are the one-turn phase advances of horizontal and vertical betatron motion.

A random 3D Langevin kick is applied to each macroion at each turn to account for the effects due to IBS. The rms amplitude of the kick is determined by the growth rate as calculated from the Piwinski formula (Appendix C) $[14,15]$. Local ion line density is used in the IBS growth rate calculations.

To implement the one-turn update due to $\mathrm{CeC}$, we first estimate how the ions are mixed from turn to turn by the synchrotron oscillation. The synchrotron period for the $\mathrm{CeC}$ experiment is about 4000 revolutions. With an rms ion bunch length of $3 \mathrm{~ns}$, the average longitudinal slippage of a typical ion in one revolution is

$$
\left\langle\Delta \tau_{1 \sigma}\right\rangle \sim \frac{4.3 \mathrm{~ns} \cdot c}{4000}=0.9 \mathrm{~mm}
$$

which is $~ 30$ times larger than the optical wavelength of the FEL amplifier $(30.5 \mu \mathrm{m})$. Consequently, no phase information is preserved after one revolution, ${ }^{8}$ and the incoherent kicks due to neighboring ions (and cooling electrons) can be implemented as a random Langevin kick, as shown in Eq. (46).

As shown in Fig. 7, the prediction from solving the Fokker-Planck equation (red-dashed line) agrees very well with that obtained from macroparticle tracking if the linear cooling force is adopted in the tracking (magenta line). It is worth noting that the peak current from solving the FokkerPlanck equation (red-dashed line) is about 1\%-2\% higher than that obtained from tracking with the linear cooling force (magenta line), which is likely due to the static diffusion coefficients assumed in the Fokker-Planck equation; meanwhile, the tracking algorithm calculates the diffusive kick from the updated bunch profile. Figure 7 also shows that the cooling effect is less pronounced if a more realistic sinusoidal cooling force is applied in the tracking, which is to be expected as ions with large synchrotron amplitude experience reduced cooling or even anticooling during the process.

\footnotetext{
${ }^{8}$ In addition to the fast mixing due to synchrotron motion, a long-term mixing has to be achieved by synchrotron tune spread or diffusion such as IBS and the shot noise in electron beam. For parameters listed in Table I, the diffusive energy kick due to electrons' shot noise alone is enough to remove the long-term correlations of the ions.
} 
During the proof of the $\mathrm{CeC}$ principle experiment in 2018, excessive noise was observed in the cooling electron beam, which made observation of the ion imprint in the electron beam unsuccessful [18]. The technique described above is also used to study the tolerance of a $\mathrm{CeC}$ system to the noise level in the electron beam. Comparing the results from macroparticle tracking with that from numerically solving the Fokker-Planck equation is illustrative for the limitation of the latter.

Figure 8 shows the current profiles of an ion bunch after $40 \mathrm{~min}$ of cooling by electrons with various noise levels. Parameters in Tables I and II are used in generating Fig. 8. Figure $8(a)$ is obtained by numerically solving Eqs. (36)(38), and Fig. 8(b) shows the results of macroparticle tracking.

While results from both approaches predict that heating due to diffusion dominates the $\mathrm{CeC}$ process once the noise in the electron beam is greater than a factor of 3 higher than its natural noise level, the peak currents of the cooled bunch in Fig. 8(b) are all lower than that shown in Fig. 8(a) due to the approximation of linear cooling force applied in the Fokker-Planck solver. On the other hand, the witness bunch (the black curve) in Fig. 8(b) has a slightly higher peak current than that in Fig. 8(a), because the IBS rate decreases with the peak current, but in the Fokker-Planck equation, the diffusion coefficient does not vary with time. Moreover, the number of particles is conserved in Fig. 8(a) as a result of using the small amplitude Hamiltonian, i.e., Eq. (5), in the Fokker-Planck equation. In tracking, particles are lost once they move out of the rf bucket and, consequently, the bunch intensity is reduced once the bunch length becomes comparable with the rf wavelength, as shown in Fig. 8(b).

\section{DISCUSSION AND SUMMARY}

In summary, we have developed a set of tools to predict the ion bunch profile in the presence of longitudinal coherent electron cooling, which include an analytical expression for vanishing diffusion coefficient, a numerical algorithm for solving Fokker-Planck equation with linear cooling force, and a macroparticle tracking code for arbitrary cooling force. In their applicable regime, we have found good agreement among these tools and used them to predict the performance of the $\mathrm{CeC}$ system in the proof of $\mathrm{CeC}$ principle experiment.

One of the insights achieved from these studies is that, for the proof of the $\mathrm{CeC}$ principle experiment, the ion bunch profile will not show any local blip during the cooling process owing to IBS or diffusion induced by neighboring particles in the cooling section. Because any stochastic cooling mechanism, including $\mathrm{CeC}$, inevitably introduces diffusive kicks due to neighboring ions, the feasibility of using high-frequency components in the beam current profile as a diagnostic tool for optimizing cooling is limited.
It is worth noting that in the kicker section of a $\mathrm{CeC}$ system, the energy kick from the electrons' density wave packet with finite width depends on the transverse location of the ion, which consequently introduces dependence of cooling rate and diffusion coefficients on the amplitude of betatron oscillation of the ion. As a 1D analysis, we have neglected such dependences. However, once the cooling force for a single pass is obtained from particle-in-cell simulation [19], it is straightforward to include such dependence in the macroparticle tracking code as described in Sec. VI.

\section{ACKNOWLEDGMENTS}

The work has greatly benefitted from discussions with V. N. Litvinenko and M. Blaskiewicz. The macroparticle tracking code is based on various subroutines written by $\mathrm{M}$. Blaskiewicz. This work was supported by Brookhaven Science Associates, LLC under Contract No. DE-AC0298CH10886 with the U.S. Department of Energy.

\section{APPENDIX A: GENERAL SOLUTION OF 1D FOKKER-PLANCK EQUATION IN THE ABSENCE OF DIFFUSION}

If the expected energy kicks from diffusion are much smaller than that from the coherent cooling kicks, i.e., $\langle D(I)\rangle \ll\langle\xi(I) I\rangle$, with the angled bracket denoting averaging over all ions, we can neglect the third term on the lhs of Eq. (1) and obtain

$$
\frac{\partial}{\partial t} \tilde{F}(I, t)-\alpha(I) \frac{\partial}{\partial I} \tilde{F}(I, t)=0,
$$

with

$$
\tilde{F}(I, t) \equiv \zeta(I) \cdot I \cdot F(I, t),
$$

and

$$
\alpha(I) \equiv I \cdot \zeta(I) .
$$

Equation (A1) can be written in the following form:

$$
\frac{d}{d t} \tilde{F}(I, t)=\frac{d I}{d t} \cdot \frac{\partial}{\partial I} \tilde{F}(I, t)+\frac{\partial}{\partial t} \tilde{F}(I, t)=0
$$

with

$$
\frac{d I}{d t}=-\alpha(I)
$$

Solving Eq. (A5) yields

$$
C=t-t_{0}+\int \frac{d I}{\alpha(I)} .
$$

Equation (A6) defines a series of contours in the $(I, t)$ plane, and each contour is specified by the value of a given 
constant $C$. If $(I, t)$ stays in the same contour, the value of $\tilde{F}(I, t)$ does not change. Thus, we obtain the general solution of Eq. (10),

$$
\tilde{F}(I, t)=G(C),
$$

with $C$ given by Eq. (A6). At $t=t_{0}$, the solution must satisfy a given initial condition,

$$
\tilde{F}\left(I, t_{0}\right)=\tilde{F}_{0}(I),
$$

and imposing the initial condition to Eq. (A7) leads to

$$
G[h(I)]=\tilde{F}_{0}(I)
$$

with

$$
h(I) \equiv \int \frac{d I}{\alpha(I)} .
$$

For any value of

$$
C=h(I),
$$

Eq. (A9) requires

$$
G(C)=\tilde{F}_{0}\left[h^{-1}(C)\right] .
$$

Inserting Eqs. (A7) and (A12) into Eq. (A2) yields the solution of Eq. (A1), which satisfies the initial condition of Eq. (A8):

$$
F(I, t)=\frac{h^{-1}(C) \zeta\left[h^{-1}(C)\right] F_{0}\left[h^{-1}(C)\right]}{\zeta(I) \cdot I} .
$$

\section{APPENDIX B: DERIVATION OF SINGLE PASS ENERGY KICK FOR FEL-BASED COHERENT ELECTRON COOLING}

Using the 1D FEL theory with high gain approximation [1], the electric field induced by a single ion at the entrance of the kicker section is

$E_{1 D}(z)=E_{p} \exp \left(-\frac{z^{2}}{2 \sigma_{w}^{2}}\right) \sin \left(k_{0} z-k_{2}^{2} z^{2}-\varphi_{0}\right)$,

where $z$ is the longitudinal location with respect to the peak of the electron density wave packet induced by the ion, $E_{p}=G \cdot E_{0}=G \cdot Z e /\left(2 \varepsilon_{0} S\right)$ is the maximal electric field induced by the electron density wave packet, $G$ is the gain of the longitudinal electric field due to FEL amplification, $S$ is the transverse area of the electron beam, $\sigma_{w}$ is the rms width of the electron density wave packet, $\varphi_{0}$ is the constant phase shift determined by the length of the FEL amplifier, and $k_{2}^{2} z^{2}$ represents the slow phase variation along the wave packet with $k_{2}^{2} z^{2} \sim\left(z / \sigma_{z \text {,rms }}\right)^{2} \ll k_{0} z$. The field observed by the $j$ th ion due to the wave packet induced by the $i^{\text {th }}$ ion is given by

$$
\begin{aligned}
E_{1 D}\left(z_{j}-\varsigma_{i}\right)= & E_{p} \exp \left[-\frac{\left(z_{j}-\varsigma_{i}\right)^{2}}{2 \sigma_{w}^{2}}\right] \sin \left[k_{0}\left(z_{j}-\varsigma_{i}\right)\right. \\
& \left.-k_{2}^{2}\left(z_{j}-\varsigma_{i}\right)^{2}-\varphi_{0}\right],
\end{aligned}
$$

where $z_{j}$ is the location of the $j$ th ion at the kicker section, and $s_{i}$ is the location of the peak of the wave packet induced by the $i$ th ion. By properly delaying the ions, the ion can be placed at

$$
z_{j}=R_{56} \cdot \delta_{j}+\Delta z_{\mathrm{sh}}+\varsigma_{j},
$$

where $\delta_{j}$ is the relative energy deviation of the $j$ th ion, $R_{56}$ is the longitudinal dispersion from the $\mathrm{CeC}$ modulator section to the kicker section, and $\Delta z_{\mathrm{sh}} \in\left(-\pi / k_{0}, \pi / k_{0}\right)$ is a small delay of the electrons introduced by the phase shifter so that for an ion with zero energy deviation, the phase of the sinusoidal function in Eq. (B2) with $i=j$ is $-\pi$, i.e.,

$$
\Delta z_{\mathrm{sh}} \approx\left[\bmod \left(\varphi_{0}, 2 \pi\right)-\pi\right] / k_{0} .
$$

Inserting Eqs. (B3) and (B4) into Eq. (B2) and assuming the electric field does not change significantly inside the kicker of length $l$, we obtain the one-turn energy kick received by the $j$ th ion in the $\mathrm{CeC}$ section:

$$
\Delta E_{j}=\Delta E_{\mathrm{coh}, j}+\Delta E_{\mathrm{inc}, j},
$$

where

$$
\Delta E_{\mathrm{coh}, j} \equiv-Z_{i} e E_{p} l \sin \left(k_{0} R_{56} \cdot \delta_{j}\right),
$$

the energy kick induced by the jth ion itself, i.e., the coherent cooling kick. The second term,

$$
\begin{aligned}
\Delta E_{\mathrm{inc}, j} \equiv & -Z_{i} e E_{p} l \sum_{i \neq j} \exp \left(-\frac{\left(\varsigma_{j}-\varsigma_{i}\right)^{2}}{2 \sigma_{w}^{2}}\right) \\
& \times \sin \left[k_{0}\left(R_{56} \delta_{j}+\varsigma_{j}-\varsigma_{i}\right)-k_{2}^{2}\left(\varsigma_{j}-\varsigma_{i}\right)^{2}\right],
\end{aligned}
$$

is the incoherent diffusive energy kick induced by all other ions. The variance of the incoherent kick is calculated as

$\left\langle\Delta E_{\mathrm{inc}, j}^{2}\right\rangle=\frac{\left(Z_{i} e E_{p} l\right)^{2}}{2}\left\langle\sum_{i \neq j} \exp \left(-\frac{\left(\varsigma_{i}-\varsigma_{j}\right)^{2}}{\sigma_{w}^{2}}\right)\right\rangle$,

where the angled bracket, $\langle\cdots\rangle$, represents ensemble average. Assuming the ion density does not vary significantly over the width of the wave packet, $\sigma_{w}$, Eq. (11) reduces to 


$$
\begin{aligned}
\left\langle\Delta E_{\text {inc }, j}^{2}\right\rangle & =\frac{\left(Z_{i} e E_{p} l\right)^{2}}{2} \int_{-\infty}^{\infty} \rho_{\text {ion }}\left(\varsigma_{i}\right) \exp \left(-\frac{\left(\varsigma_{i}-\varsigma_{j}\right)^{2}}{\sigma_{w}^{2}}\right) d \varsigma_{i} \\
& \approx \frac{\left(Z_{i} e E_{p} l\right)^{2}}{2} \sqrt{\pi} \rho_{\text {ion }}\left(z_{j}\right) \sigma_{w},
\end{aligned}
$$

where $\rho_{\text {ion }}\left(z_{j}\right)$ is the local line number density of ions around the $j$ th ion. The energy kicks in Eq. (B5) can be approximated as the summation of the coherent kick, as described in Eq. (B6) and a random kick with the rms amplitude described by Eq. (B9):

$\Delta E_{j, N} \approx-Z_{i} e E_{p} l \sin \left(k_{0} R_{56} \cdot \delta_{j}\right)+\sqrt{\frac{\left\langle\Delta E_{\mathrm{inc}, j}^{2}\right\rangle}{\left\langle X^{2}\right\rangle}} \cdot X_{j, N}$,

where $X_{j, N}$ is a random number determining the incoherent kick acting on the $j$ th ion at the $N^{\text {th }}$ turn, and $\left\langle X^{2}\right\rangle$ is the variance of $X_{j, N}$. For instance, if $X_{j, N}$ is a uniformly distributed random number from -1 to 1 , its variance is

$$
\left\langle X^{2}\right\rangle=\frac{1}{2} \int_{-1}^{1} X^{2} d X=\frac{1}{3},
$$

and Eq. (B10) becomes

$$
\begin{aligned}
\Delta E_{j, N} \approx & -Z_{i} e E_{p} l \sin \left(k_{0} R_{56} \cdot \delta_{j}\right) \\
& +Z_{i} e E_{p} l \sqrt{\frac{3}{2} \sqrt{\pi} \rho_{\text {ion }}\left(z_{j}\right) \sigma_{w}} \cdot X_{j, N} .
\end{aligned}
$$

Following a similar derivation, the incoherent kick due to shot noise from cooling electrons is derived as

$$
\Delta E_{j, N}^{e} \approx e E_{p} l \sqrt{\frac{3}{2} \sqrt{\pi} \rho_{e}\left(z_{j}\right) \sigma_{w}} \cdot X_{j, N}
$$

where $\rho_{e}\left(z_{j}\right)$ is the line number density of the electrons at location $z_{j}$.

\section{APPENDIX C: ENERGY KICK DUE TO INTRABEAM SCATTERING [20]}

Here, we derive the Langevin kicks to the energy of each ion such that the overall growth rate of the longitudinal emittance of the ion beam is given by the value as calculated from the Piwinski formula [14,15,21]. For an ion at its nth circulation with longitudinal coordinate $\left(\phi_{n}, P_{n}\right)$, after receiving momentum kick $\delta_{P}$ due to IBS, its action as defined in Eq. (5) increases by

$$
\Delta I=I_{n+1}-I_{n}=\frac{1}{2}\left[\left(P_{n}+\delta_{P}\right)^{2}-P_{n}^{2}\right]=P_{n} \delta_{P}+\frac{1}{2} \delta_{P}^{2} .
$$

Assuming $\delta_{P}$ is uncorrelated with $P_{n}$, the average increase in the action of the ion is given by the ensemble average of Eq. (C1):

$$
\langle\Delta I\rangle=\frac{\left\langle\delta_{P}^{2}(\phi)\right\rangle}{2} .
$$

Assuming

$$
\left\langle\delta_{P}^{2}(\phi)\right\rangle=\delta_{\max }^{2} \cdot \exp \left(-\frac{\phi^{2}}{2 \sigma_{\phi}^{2}}\right)
$$

the average increase in the action of the ion beam is

$$
\begin{aligned}
\langle\Delta I\rangle= & \frac{\left\langle\delta_{P}^{2}\right\rangle}{2}=\frac{1}{4 \pi I_{\text {ion }}} \int_{-\infty}^{\infty} \exp \left(-\frac{I}{I_{\text {ion }}}\right) \delta_{\max }^{2} \\
& \cdot \exp \left(-\frac{\phi^{2}}{2 \sigma_{\phi}^{2}}\right) d P d \phi=\frac{\delta_{\max }^{2}}{2 \sqrt{2}} .
\end{aligned}
$$

The average action of the beam before the IBS kick is

$$
\langle I\rangle=\frac{1}{4 \pi I_{\text {ion }}} \int_{-\infty}^{\infty} \exp \left(-\frac{I}{I_{\text {ion }}}\right)\left(P^{2}+\phi^{2}\right) d P d \phi=I_{\text {ion }}=\sigma_{P}^{2} .
$$

The IBS rise time for the beam energy spread is

$$
\frac{1}{T_{\mathrm{IBS}}}=\frac{1}{T_{\mathrm{rev}}} \frac{\langle\Delta I\rangle}{2\langle I\rangle} .
$$

Inserting Eqs. (C4) and (C5) into Eq. (C6) yields

$$
\delta_{\max }^{2}=\frac{4 \sqrt{2} \sigma_{P}^{2}}{T_{\mathrm{IBS}}} T_{\mathrm{rev}} .
$$

With the line density of the beam taken as

$$
\rho(\phi)=\frac{1}{\sqrt{2 \pi} \sigma_{\phi}} \exp \left(-\frac{\phi^{2}}{2 \sigma_{\phi}^{2}}\right),
$$

the standard deviation of the kick should be

$$
\begin{aligned}
\left\langle\delta_{P}^{2}(\phi)\right\rangle & =\delta_{\max }^{2} \cdot \exp \left(-\frac{\phi^{2}}{2 \sigma_{\phi}^{2}}\right)=\delta_{\max }^{2} \frac{\rho(\phi)}{\rho_{\max }} \\
& =8 \sqrt{\pi} \sigma_{\phi} \sigma_{P}^{2} \frac{T_{\mathrm{rev}}}{T_{\mathrm{IBS}}} \rho(\phi) .
\end{aligned}
$$

If we take random number $X_{i}$ uniformly distributed around -1 to 1 , the amplitude in front of the random number should be

$$
d_{\mathrm{IBS}}=\left[3\left\langle\delta_{P}^{2}(\phi)\right\rangle\right]^{1 / 2}=\left[24 \sqrt{\pi} \sigma_{\phi} \sigma_{P}^{2} \frac{T_{\mathrm{rev}}}{T_{\mathrm{IBS}}} \rho(\phi)\right]^{1 / 2}
$$


[1] C. Montag et al., eRHIC design overview, in The 10th International Particle Accelerator Conference, Melbourne, Australia, 2019 (JACoW Publishing, Geneva, Switzerland, 2019), pp. 45-48.

[2] Y. Zhang, JLEIC: A high luminosity polarized electron-ion collider at jefferson lab, in The 10th International Particle Accelerator Conference, Melbourne, Australia, 2019, (JACoW Publishing, Geneva, Switzerland, 2019), pp. 19161919.

[3] V. N. Litvinenko and Y. S. Derbenev, Coherent Electron Cooling, Phys. Rev. Lett. 102, 114801 (2009).

[4] D. Ratner, Microbunched Electron Cooling for HighEnergy Hadron Beams, Phys. Rev. Lett. 111, 084802 (2013).

[5] G. Stupakov, Cooling rate for microbunched electron cooling without amplification, Phys. Rev. Accel. Beams 21, 114402 (2018).

[6] G. Stupakov and P. Baxevanis, Microbunched electron cooling with amplification cascades, Phys. Rev. Accel. Beams 22, 034401 (2019).

[7] Y.S. Derbenev, Coherent electron Cooling, in The 7th All Union Conference on Charged Particle Accelerators, Dubna, USSR, 1980 (Joint Inst. Nucl. Res., Dubna, Soviet Union, 1981).

[8] G. Wang, M. Blaskiewicz, and V. Litvinenko, Analytical studies of ion beam evolution under coherent electron cooling, in The 7th International Particle Accelerator Conference, Busan, Korea, 2016 (JACoW Publishing, Geneva, Switzerland, 2016), pp. 1247-1249.

[9] G. Wang, M. Blaskiewicz, and V. Litvinenko, Simulation of ion beam under coherent electron cooling, in The 7th International Particle Accelerator Conference, Busan, Korea, 2016 (JACoW Publishing, Geneva, Switzerland, 2016), pp. 1243-1246.

[10] S. Chattopadhyay, Theory of bunched beam stochastic cooling, IEEE Trans. Nucl. Sci. 30, 2649 (1984).

[11] J. Wei and A. Ruggiero, in Proceedings of the 1991 Particle Accelerator Conference, San Francisco, CA, 1991 (IEEE, New York, 1991), pp. 1869-1871.
[12] S.-Y. Lee, Accelerator Physics (World Scientific, Singapore, 2004).

[13] W. H. Press, S. A. Teukolsky, W. T. Vetterling, and B. P. Flannery, Numerical Recipes in Fortran: The Art of Scientific Computing, 2nd ed. (The Press Syndicate of the University of Cambridge, New York, 1992).

[14] A. Piwinski, Intra-beam Scattering, in the 9th International Conference on High-Energy Accelerators, Stanford, California (SLAC, Stanford, California, 1974), pp. 405409.

[15] A. Piwinski, J. D. Bjorken, and S. K. Mtingwa, Wilson Prize article: Reflections on our experiences with developing the theory of intrabeam scattering, Phys. Rev. Accel. Beams 21, 114801 (2018).

[16] H. Wiedemann, Particle Accelerator Physics (Springer, New York, 2015).

[17] G. Wang, Y. Jing, J. Ma, and V. Litvinenko, Electron beam requirements for coherent electron cooling FEL system, in The 38th International Free Electron Laser Conference, Santa Fe, NM, USA (JACoW Publishing, Geneva, Switzerland, 2018), pp. 319-321.

[18] V. Litvinenko et al., Coherent electron cooling (CeC) experiment at RHIC: Status and plans, in The 10th International Particle Accelerator Conference, Melbourne, Australia (JACoW Publishing, Geneva, Switzerland, 2019), pp. 2101-2104.

[19] J. Ma, G. Wang, and V. Litvinenko, 3d Start-to-end simulations of the coherent electron cooling, in The 10th International Particle Accelerator Conference, Melbourne, Australia (JACoW Publishing, Geneva, Switzerland, 2019), pp. 3329-3332.

[20] M. Blaskiewicz (private communication).

[21] A. W. Chao and M. Tigner, Handbook of Accelerator Physics and Engineering (World Scientific Publishing Co. Pte. Ltd, New Jersey, 1998). 